\title{
Dynamics of Changes in Concentration of Inflammatory and Destructive Biomarkers at Various Stages of Atherosclerotic Plaque Development
}

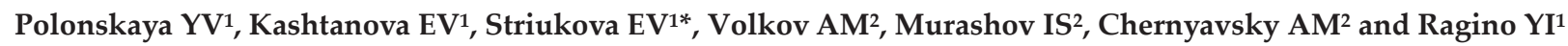 \\ ${ }^{*}$ Research Institute of Internal and Preventive Medicine - Branch of the Institute of Cytology and Genetics, Siberian Branch of Russian Academy of \\ Sciences, Russia \\ 2E.N. Meshalkin Novosibirsk Research Institute of Circulation Pathology Ministry of Healthcare of Russia, Russia \\ *Correspondence to: Striukova EV, Research Institute of Internal and Preventive Medicine - Branch of the Institute of Cytology and Genetics, Siberian Branch of \\ Russian Academy of Sciences, Russia, Novosibirsk, st. B.Bogatkova 175/1, 630089; E-mail: stryukova.j@mail.ru
}

Received: February 10, 2018; Accepted: February 20, 2018; Published: February 23, 2018;

\begin{abstract}
\section{Goal of research}

To examine factors and mechanisms of occurrence and progression of atherosclerotic focus and determine key among them in development of atherosclerotic unstable plaque.
\end{abstract}

\section{Materials and methods}

Research included 130 men with coronary atherosclerosis to whom during surgery was performed endarterectomy from coronary artery/arteries. Every substance after endarterectomy was divided into fragments for histological and biochemical examination. Through enzyme-linked immunoassay with the use of standard set (ELISAs) in samples homogenates were identified levels of TNF- $\alpha$, IL-1- $\beta$, IL-1- PA, IL-2, IL-6, IL-8, hsCRP, TIMP-1, MMP-9, MMP-3, MMP-1, MMP-7, MCP-1, EMAP-II. Statistic processing of the results was made with the use of software program SPSS for Windows.

Results

As atherosclerotic focus develops to the stage of stable plaque activity of TIMP-1 decreases in it and increase levels of MMP-7, MMP-1, MMP-9 that reflexes intense destruction processes of connective-tissue matrix in unstable plaque, in its fibrous cover. Inflammatory activity in unstable plaque manifest in increased IL-6, IL-8 and CRP levels. There are also highest levels of MCP-1and EMAP-II in them.

At the stage of stable plaque manifestations of destructive activity in the form of increased MMP-3 levels and reduced concentration of TIMP-1 statistically significantly depend on inflammatory oxidative changes, whose key factors are increased TNF- $\alpha$ levels and reduced IL-1-RA levels.

\section{Conclusion}

During process of atherosclerotic focus development to the stage of unstable plaque acute inflammatory process activity and tissue damage reactions increase in it, activate processes of connective-tissue matrix and plaque fibrous cover destruction that leads to its thinning, excision, bursting/breakage.

As atherosclerotic focus develops at the stage of stable plaque and at the stage of unstable plaque manifestations of destructive activity significantly depend on inflammatory oxidative changes various at every stage.

Key words: coronary atherosclerosis, atherosclerotic plaque, metalloproteinase, inflammatory marker

\section{Introduction}

Contemporary views on unstable atherosclerotic plaque formation key mechanisms are controversial. Inflammatory and destructive process plays important role in unstable atherosclerotic plaque development. [1-3]. Many cytokines such as TNF- $\alpha$, IL-1, IL-2, IL-3, IL-6, IL-8, CXCL8, IL-10, IL-12, IL-18, IFN- $\gamma$ are to be found in vessels with risk of atherosclerosis. In hyperlipidemia macrophages produce TNF- $\alpha$, IL-1, IL-6, IL-12 and IL-18 and also anti-inflammatory cytokines IL-10 and TGF- $\beta$. Besides anti-inflammatory cytokines and chemoattractants macrophages produce matrix metalloproteinases (MMP) that cause degradation of extracellular matrix. [4]. Among them MMP-1, MMP-13 collagenases break down fibrillar collagen types I and III, MMP-2, MMP-9 gelatinises and MMP-3 stromelysin break down adhesion molecules, nonfibrillar forms of collagen and proteoglycans. Tissue inhibitor of metalloproteinases type 1 (TIMP1) inhibits MMP-1, MMP-3, MMP-9 and in connection with active catalytic core blocks its activity forming non-covalent complexes. (for instance, complex TIMP-1/ MMP-3) [5,6]. 
To date investigation results of the inflammatory and destructive, oxidative, endothelial dysfunctional processes activity in dynamics of atherosclerotic focus stage development up to unstable plaque are not numerous, so that studies are relevant in this area. Some questions concerning destabilization of plaque mechanisms, characteristics of correlations between many factors, inducing this process remain unclear. That implies difficulties in elaboration of effective preventive and therapeutic approaches to stabilization of atherosclerotic focuses.

\section{Materials and methods}

Research included men aged 49 to 79 with verified coronary atherosclerosis, stable effort angina functional class II-III (without acute coronary syndrome) that were admitted to E.N. Meshalkin Novosibirsk Research Institute of Circulation Pathology Ministry of Healthcare of Russia, Novosibirsk for coronary bypass surgery. Exclusion criteria were myocardial infarction not older than 6 month, acute inflammatory diseases, acute exacerbation of chronic diseases, active hepatic disorders, chronic renal diseases and oncology diseases. Every patient filled out Informed consent form for taking part in research. During surgery in 130 patients was performed endarterectomy from coronary artery/arteries for intraoperative indications. Every substance after endarterectomy containing intimamedia complex from coronary arteries was both transversally and longitudinally symmetrically divided into 3-5 fragments. As the result was obtained 415 samples for histological and biochemical examination.

Histological analysis of collected intima-media samples from coronary arteries was performed after macroscopic samples description (prevalence of plaque, degree of artery opening stenosis formation, hemorrhaging in the structures of plaque, calcification focuses and thrombi) and after standard hematoxylin-eosin and van Gieson's staining.

From 415 samples 32 cases were identified as intact intima tissue, 41 as lipid spot/streak, 72 as stable immature atheromatosis plaque, 105 as stable atheromatosis plaque with fibrosis/calcinosis, 165 as unstable atheromatosis plaque with tendency to ulceration and rupture. Unstable plaques were identified by this criteria: damaged plaque with fibrous cover less than $65 \mu \mathrm{m}$ in thickness, infiltrated with macrophages and T cells (lymphocytes) (more than 25 cells per high power field $0,3 \mathrm{~mm}$ ) with large lipidic nucleus $(>40 \%)$

Through enzyme-linked immunoassay with the use of standard set (ELISAs) in samples homogenates were identified levels of TNF- $\alpha$ (Biosource), IL-1- $\beta$ and IL-1- PA (Biosource), IL-2, IL-6, IL-8 (Cytimmune), hsCRP (Biomerica), TIMP-1(Biosource), MMP9 (RD), MMP-3 (Biosource), MMP-1 (RayBiotech), MMP-7 (BCM Diagnostics panel), chemoattractants MCP-1 (BenderMedSystems), EMAP-II (Biosource). Control serums, sourced with ELISAs panels, with high and low level of test indexes were used as control.

Statistic processing of the results was made with the use of software program SPSS for Windows. For every index were calculated straight average $(M)$, standard deviation $(\sigma)$, standard error of the mean $(m)$, median (Me), highest and lowest value in relation to distribution type. Significance of differences between mean values was estimated with the use of Student t-test (for attributes with Gaussian distribution model) or Mann-Whitney U test. Correlation relationships were estimated with the use of Spearman's criteria. Multiple comparisons between groups were made though analysis of variance (One-Way-ANOVA) with the use of Dunnett's test for multiple comparisons. Associative links were estimated within the system of linear regression analysis, multivariate regression analysis in GLM. Statistical significance criteria was $\mathrm{p}<0,05$.

\section{Results and discussion}

For appraisal of inflammatory activity in homogenates of samples in various stages of atherosclerotic focuses development were investigated levels of some proinflammatory cytokines, CRP and chemoattractants. (Table 1).

The lowest level of IL-1-RA was noticed in stable immature and fibrous atherosclerotic plaques (Table 2), lower than in intact intima tissue and lipid spots. Level of IL-1-RA in unstable plaques was no different than in intact intima tissue and lipid spots and was even 1,6 times higher than in immature stable atherosclerotic plaques. Whereas IL-1-RA is the competitive antagonist of IL-1 in interacting process with specific receptor to IL-1, obtained results attest that there are no increased levels of IL-1 in unstable plaques. And over against this results reflect increased levels of IL-1 in the earlier stage of atheroma development - immature stable atherosclerotic plaque stage.

While analyzing the TNF- $\alpha$ levels was also noticed that there were stable plaques, where levels of test item were different. Level of TNF- $\alpha$ in immature stable and fibrotic plaques was more than 2 times higher in comparison with intact intima tissue, lipid spots and unstable plaques. Considering that withdrawal of biomaterial was performed in men without ACS it is possible to suggest that activity of IL- 1 and TNF- $\alpha$ is increased and plays one of key roles in development of atherosclerotic focus at the stage of stable plaque (immature or fibrotic) by inducing proliferation of T- cells, smooth muscle cells and expression of adhesion molecules $[7,8]$, but isn't dominant at the stage of unstable plaque.

Examination of proinflammatory cytokines IL-2, IL-6 and IL-8 showed predominance of activity in unstable plaques. Although IL-2 level found out only upward trend, no significant contrast was identified, IL-6 and IL-8 levels were considerably higher in unstable plaques. Consequently IL-6 (inductor of acute phase reaction of inflammatory response and tissue damage [9]) level in unstable plaques were 2,7 times higher than in fibrous plaques and 4 times higher than in intact tissue, lipid spots, stable immature plaques. In unstable plaques IL-8 level also proved to be highest, higher than in intact tissue, lipid spots, stable immature (in 7,8; 6,5 and 5,2 times respectively) and fibrous ( 1,7 times) plaques. Beyond that IL- 6 and IL-8 levels were higher in stable and fibrous plaques in comparison with intact intima, lipid spots and stable immature plaques. IL-1 and TNF- $\alpha$ are known to stimulate secretion of IL- 8 by macrophages and endothelial cells therefore it can be assumed that this is predominance of activity of IL- 1 and TNF- $\alpha$ at earlier stages of atherosclerotic focus development that potentiate subsequent increase of IL- 8 secretion and is evident at the stage of unstable plaque. 
Striukova EV (2018) Dynamics of Changes in Concentration of Inflammatory and Destructive Biomarkers at Various Stages of Atherosclerotic Plaque Development

Table 1. Proinflammatory cytokines and chemoattractants concentration in atherosclerotic focuses at various stages of atherosclerotic plaque development ( $\pm \sigma$ )

\begin{tabular}{|c|c|c|c|c|c|}
\hline Homogenates & 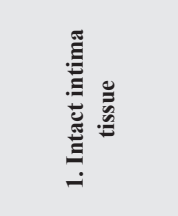 & 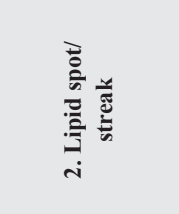 & 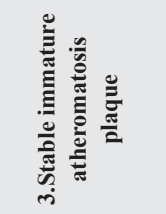 & 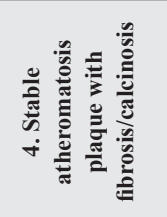 & 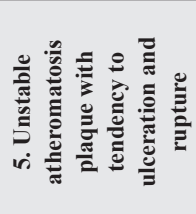 \\
\hline $\begin{array}{c}\text { IL-1-RA, } \\
\mathrm{pg} / \mathrm{mg} \text { of protein }\end{array}$ & $1255.1 \pm 106.1 * \#$ & $1120.5 \pm 100.7^{* \#}$ & $652.5 \pm 61.3$ & $805.1 \pm 72.2$ & $1044.9 \pm 93.5^{*}$ \\
\hline $\begin{array}{c}\text { TNF- } \alpha, \\
\mathrm{pg} / \mathrm{mg} \text { of protein }\end{array}$ & $2.6 \pm 0.4 * \#$ & $4.3 \pm 0.5 * \#$ & $8.2 \pm 0.7$ & $6.5 \pm 0.5$ & $3.1 \pm 0.4 * \#$ \\
\hline $\begin{array}{c}\text { IL-6, } \\
\mathrm{ng} / \mathrm{mg} \text { of protein }\end{array}$ & $3.8 \pm 0.4 \#^{\wedge}$ & $3.6 \pm 0.4 \#^{\wedge}$ & $3.2 \pm 0.3 \#^{\wedge}$ & $5.7 \pm 0.5^{\wedge}$ & $15.9 \pm 1.1 \#$ \\
\hline $\begin{array}{c}\text { IL-2, } \\
\mathrm{pg} / \mathrm{mg} \text { of protein }\end{array}$ & $16.7 \pm 2.2$ & $17.8 \pm 2.5$ & $17.4 \pm 3.5$ & $19.9 \pm 2.3$ & $21.9 \pm 3.4$ \\
\hline $\begin{array}{c}\text { IL- } 8 \\
\mathrm{pg} / \mathrm{mg} \text { of protein }\end{array}$ & $4.7 \pm 0.5 \#^{\wedge}$ & $5.7 \pm 0.5 \#^{\wedge}$ & $7.1 \pm 0.6 \#^{\wedge}$ & $22.2 \pm 2.9^{\wedge}$ & $37.1 \pm 4.8 \#$ \\
\hline $\begin{array}{c}\text { MCP- } 1 \\
\mathrm{pg} / \mathrm{mg} \text { of protein }\end{array}$ & $208.9 \pm 21.0 \#^{\wedge}$ & $218.9 \pm 20.5 \#^{\wedge}$ & $208.4 \pm 19.6 \#^{\wedge}$ & $347.1 \pm 31.0$ & $401.7 \pm 39.8$ \\
\hline $\begin{array}{c}\text { EMAP-II, } \\
\mathrm{pg} / \mathrm{mg} \text { of protein }\end{array}$ & $510.1 \pm 46.1^{*} \#^{\wedge}$ & $901.8 \pm 76.6^{*} \#^{\wedge}$ & $1318.6 \pm 115.0^{\wedge}$ & $1475.2 \pm 131.1^{\wedge}$ & $2007.8 \pm 189.5$ \\
\hline $\begin{array}{c}\text { CRP, } \\
\mathrm{ng} / \mathrm{mg} \text { of protein }\end{array}$ & $7.0 \pm 1.0 \#^{\wedge}$ & $11.2 \pm 1.6 \#^{\wedge}$ & $12.2 \pm 1.0 \#^{\wedge}$ & $22.7 \pm 2.6$ & $28.7 \pm 2.1$ \\
\hline
\end{tabular}

Footnote: *- In comparison with atheromatosis plaque with fibrosis/calcinosis $(\mathrm{p}<0,05)$; \# - In comparison with stable plaque $(\mathrm{p}<0,05)$; ${ }^{-}$In comparison with unstable plaque $(\mathrm{p}<0,05)$

Table 2. Matrix metalloproteinases concentration in atherosclerotic focuses at various stages of atherosclerotic plaque development (M $\pm \sigma$ )

\begin{tabular}{|c|c|c|c|c|c|}
\hline 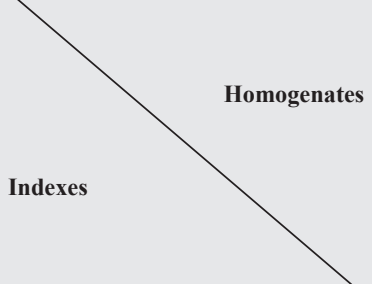 & 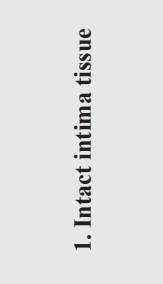 & 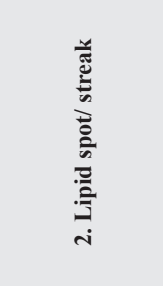 & 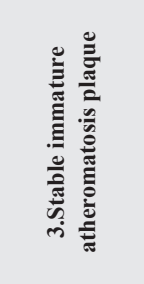 & 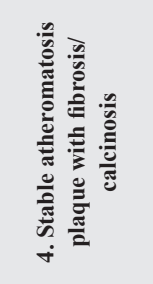 & 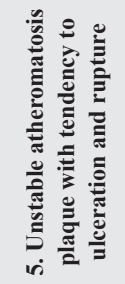 \\
\hline $\begin{array}{c}\text { TIMP-1, } \\
\mathrm{ng} / \mathrm{mg} \text { of protein }\end{array}$ & $241.2 \pm 26.2^{*} \#^{\wedge}$ & $203.4 \pm 20.1^{*} \#^{\wedge}$ & $92.5 \pm 8.8$ & $120.1 \pm 19.9$ & $117.2 \pm 11.3$ \\
\hline $\begin{array}{c}\text { MMP-3, } \\
\text { ng/mg of protein }\end{array}$ & $2.9 \pm 0.2^{*}$ & $3.0 \pm 0.4$ & $4.6 \pm 0.5$ & $3.2 \pm 0.3$ & $2.4 \pm 0.2 *$ \\
\hline $\begin{array}{c}\text { MMP-7, } \\
\text { ng/mg of protein }\end{array}$ & $0.53 \pm 0.05^{\wedge}$ & $1.53 \pm 0.1^{\wedge}$ & $1.74 \pm 0.1$ & $1.65 \pm 0.1^{\wedge}$ & $2.32 \pm 0.2$ \\
\hline $\begin{array}{c}\text { MMP-9, } \\
\text { ng/mg of protein }\end{array}$ & $2.3 \pm 0.3^{\wedge}$ & $2.6 \pm 0.4^{\wedge}$ & $2.9 \pm 0.4^{\wedge}$ & $3.8 \pm 0.4^{\wedge}$ & $6.2 \pm 0.6$ \\
\hline $\begin{array}{c}\text { MMP-1, } \\
\mathrm{pg} / \mathrm{mg} \text { of protein }\end{array}$ & $75.98 \pm 22.6^{\wedge}$ & $91.45 \pm 27.6^{\wedge}$ & $104.6 \pm 21.4^{\wedge}$ & $111.33 \pm 23.7^{\wedge}$ & $208.6 \pm 41.8$ \\
\hline
\end{tabular}

Footnote: *- In comparison with atheromatosis plaque with fibrosis/calcinosis $(\mathrm{p}<0,05)$; \# - In comparison with stable plaque ( $<<0,05)$; ^ - In comparison with unstable plaque ( $<0,05)$

MCP-1 expressed by macrophages in response to affecting by such cytokines as TNF- $\alpha$, IL-1-beta and IL-6 is monocyte and T-cell specific chemoattractant. [10,11]. The study on content of MCP1 at different stages of atherosclerotic focus development showed its gradual increase from intact intima tissue to unstable plaque. In unstable plaques MCP-1 levels were highest, higher than in intact intima, lipid spots and stable immature plaques (in 1,9 times). MCP-1 levels in stable fibrous plaques have been also higher in comparison with intact intima, lipid spots and stable immature plaques.
Endothelial-Monocyte Activating Polypeptide II (EMAP-II) released by macrophages and T-cells is proinflammatory cytokine and chemoattractant that induces formation of procoagulaitive tissue factor at the surface of endotheliocytes, neutrophil activation, apoptosis, inhibition of arteria wall intima/media neovascularization. The study on content of EMAP-II showed its gradual increase as atherosclerotic focus develops and largest predominance in unstable plaques (in 3.9 times higher than in intact intima; in 2,2 times higher than in lipid spots; in 1,5 times higher than in stable immature plaques 
and in 1,4 times higher than in fibrous plaques). It should be noted that EMAP-II level was also higher in stable immature and fibrous plaques in comparison with intact intima and lipid spots.

The study on content of C-reactive protein levels at different stages of atherosclerotic focus development demonstrated its gradual increase from intact intima tissue to unstable plaque. In unstable plaques samples $\mathrm{C}$-reactive protein levels were higher than in samples of intact tissue, lipid spots and stable immature plaques in 4,1;2,5 and 2,3 times respectively. C-reactive protein levels in fibrous calcareous plaques samples were also higher in comparison with samples of intact intima tissue, lipid spots and stable immature plaques in 3,2; 2,0 and 1,8 times respectively. Therefore results obtained demonstrated inflammatory activity in unstable plaques manifested in increased levels of IL-6, IL-8 and CRP. Also MCP-1and EMAP-II levels turned out highest in unstable plaques.

Furthermore we examined variations in metalloproteinases and its inhibitor levels as markers of destructive process activity at various stages of gradual atherosclerotic focus development to the stage of unstable plaque.

TIMP-1 levels in stable immature and fibrous plaques and in unstable plaques were lower than in intact intima and lipid spots (in 1,7 times). However there was no significant difference between stable and unstable plaques. TIMP-1 is MMP-1, MMP-3, MMP-9 inhibitor. Connecting with active catalytic center of MMP TIMP-1 block their activity forming non-covalent complexes (for example, TIMP-1/ MMP-3 complex). [12,13]. TIMP-1 activity in its turn can be inhibited by increased level of IL-8 $[14,13]$, that conform to results obtained on IL-8 levels in plaques.

Reduced concentration of TIMP-1 in formed atherosclerotic plaques (stable and unstable) demonstrates the greater potential opportunity of destructive MMP enzymatic activity in them. However expected results were not received relating to MMP-3. MMP-3 level (stromelysin destructing elastin, proteoglycans, fibronectin etc. [13]) turned out higher only in stable immature plaques, at that in comparison both with intact intima and unstable plaques (in 2,4 times) that demonstrate that there was no increased activity of MMP3 in unstable plaques even with reduced activity of TIMP-1 in them.

The study on content of MMP-7 (matrilysin destructing proteoglycans, fibronectin, laminin, versican etc. [15,13]), that besides intrinsic activity is activator of pro-MMP-9 and MMP-9 itself ( gelatinase hydrolyzing collagen of basal membranes [12,13]), contrariwise revealed their evident increase in unstable plaques rather than anywhere else in comparison with intact intima, lipid spots and stable immature plaques (in 1,4 and 1.6 times relatively).

During study on MMP-1, destructing collagen fibers was revealed its rise in 1,9 times $(\mathrm{p}<0,05)$ in unstable atherosclerotic focuses in comparison with stable and in 2,7 times in comparison with intact intima tissue.

Therefore as atherosclerotic focus develops to the stage of unstable plaque levels of inflammatory cytokines IL-6 and IL-8, chemoattractants MCP-1 and EMAP-II increase in it. That points to intensification of acute inflammatory process activity and reactions of tissue damage in unstable plaques that is accompanied by increased chemotaxis of monocytes, neutrophils and T-cells. As atherosclerotic focus develops to the stage of unstable plaque activity of TIMP-1 decrease in it and increase levels of MMP-7, MMP-1and MMP-9. That reflects intense destruction processes of connective-tissue matrix in unstable plaque, in its fibrous cover that leads to its thinning, excision bursting/breakage.

Carried out correlation analysis revealed significant linkage between test item of destructive changes and inflammatory oxidative changes in atherosclerotic focuses.

Therefore here are shown significant correlations for MMP-9 levels not only with levels of TIMP-1, MMP-3, MCP-1 $(p<0,01)$, but also with levels of IL-1-PA $(p<0,01)$, TNF- $\alpha(p<0,05)$, IL-6, IL-8 $(\mathrm{p}<0,01)$ and CRP $(p<0,01)$. Was revealed significant average correlation dependence between MMP-3 level and TNF- $\alpha(p<0,01)$. Significant negative correlation was shown for TIMP- 1 with such parameters of activity as TNF- $\alpha$, IL-6 $(p<0,05)$, IL-8 $(p<0,01)$ and direct correlation with IL-1-RA level $(p<0,01)$. Were revealed significant direct correlation dependences between lipid peroxidation output levels and MMP-9 $(p<0,01)$, MMP-3 $(p<0,05)$ and negative correlation with TIMP-1 $(p<0,01)$. As expected were revealed correlation dependences between CRP levels and cytokines TNF- $\alpha$, IL-6 $(p<0,05)$ and IL-8 $(p<0,01)$ in atherosclerotic focuses.

Certainty of dependences between destructive changes activity (matrix MMP levels and its tissue inhibitor) and inflammatory oxidative processes in atherosclerotic plaques was estimated through regression and multivariant GLM model (General Linear Model) analysis. Destructive activity indexes were included in model in the capacity of dependant variables while spectrum of the investigated inflammatory oxidative indexes in the capacity of independent variables (explanatory variables). Was revealed statistically significant dependence MMP-9 level on IL-6, IL-8 and IL-18 levels (standardized rate $\mathrm{B}=0,343 ; 0,329$ and 0,372 relatively, $p<0,05)$ corresponding to characteristics of revealed inflammatory oxidative changes in unstable plaques. Was shown dependence MMP-3 levels on TNF- $a$ levels $(B=0,517, p<0,001)$ corresponding to characteristics of revealed inflammatory oxidative changes in stable plaques. Were also revealed dependences TIMP-1 level on IL-1-RA levels $(B=0,677, p<0,01)$, that corroborate characteristics of revealed inflammatory oxidative changes in stable plaques.

Therefore as atherosclerotic focus develops at the stage of stable plaque manifestations of destructive activity in the form of increased MMP-3 levels and reduced concentration of TIMP-1 revealed statistically significantly dependence on inflammatory oxidative changes, which key factors are increased TNF- $\alpha$ levels and reduced IL-1-RA levels. Further as atherosclerotic focus develops to the stage of unstable plaque manifestations of destructive activity in the form of increased MMP-7, MMP-1, MMP-9 levels depend on inflammatory changes which key factors are increased IL-6, IL-8, MCP-1 and EMAP-II levels. 


\section{Conclusion}

During process of atherosclerotic focus stage development to the stage of unstable plaque inflammatory cytokines and chemoattractants increase in it that reflects intensification of acute inflammatory process activity, tissue damage reactions in unstable plaque. As atherosclerotic focus develops to the stage of unstable plaque activity of matrix metalloproteinases inhibitor decreases and significantly increase levels of destructive enzymes that reflects activation of destruction processes of connective-tissue matrix in unstable plaque, fibrous cover of the plaque that leads to its thinning, excision bursting/breakage.

As atherosclerotic focus develops at the stage of stable plaque and at the stage of unstable plaque manifestations of destructive activity significantly depend on inflammatory oxidative changes various at every stage.

\section{Conflict of interest}

None declared.

\section{Acknowledgements}

The study was supported by Federal Agency for scientific organizations program for support the bio resource collections.

\section{References}

1. Sakakura K, Nakano M, Otsuka F, Ladich E, Kolodgie FD, et al. (2013) Pathophysiology of atherosclerosis plaque progression. Heart Lung Circ 22: 399411. [crossref]

2. Libby P, Okamoto Y, Rocha VZ, Folco E (2010) Inflammation in atherosclerosis: transition from theory to practice. Circ J 74: 213-220. [crossref]

3. Moreno PR (2010) Vulnerable plaque: definition, diagnosis, and treatment. Cardiol Clin 28: 1-30. [crossref]

4. Pigarevsky PV, Snegova V, Maltseva SV, Davydova NG (2015) T-cells and macrophages in unstable atherosclerotic affects in human. Cytokines and inflammation Journal.

5. Ries C (2014) Cytokine functions of TIMP-1. Cell Mol Life Sci 71: 659-672. [crossref]

6. Ambrose JA, Srikanth S (2010) Vulnerable plaques and patients: improving prediction of future coronary events. Am J Med 123: 10-16.

7. Hansson GK, Libby P, Tabas I (2015) Inflammation and plaque vulnerability. Journal of internal medicine 5: 483-493.

8. Wohlschlaeger J, et al. (2015) Coronary atherosclerosis and progression to unstable plaques: Histomorphological and molecular aspects. Herz 6: 837-844.

9. Hartman J, Frishman WH (2014) Inflammation and atherosclerosis: a review of the role of interleukin- 6 in the development of atherosclerosis and the potential for targeted drug therapy. Cardiology in review 3: 147-151.

10. Schiopu A. et al. (2016) Associations Between Macrophage Colony-Stimulating Factor and Monocyte Chemotactic Protein 1 in Plasma and First-Time Coronary Events: A Nested Case-Control Study. Journal of the American Heart Association e002851.

11. Zernecke A, Weber C (2014) Chemokines in Atherosclerosis. Arteriosclerosis, thrombosis, and vascular 4: 742-750.

12. Wang M, et al. (2015) Matrix metalloproteinases promote arterial remodeling in aging, hypertension, and atherosclerosis. Hypertension 4: 698-703.

13. Newby AC (2016) Metalloproteinase production from macrophages-a perfect storm leading to atherosclerotic plaque rupture and myocardial infarction. Experimental physiology 1: 1327-1337.

14. Silvello D. et al. (2014) Serum levels and polymorphisms of matrix metalloproteinases (MMPs) in carotid artery atherosclerosis: higher MMP-9 levels are associated with plaque vulnerability. Biomarkers 19: 49-55.

\section{Citation:}

Polonskaya YV, Kashtanova EV, Striukova EV, Volkov AM, Murashov IS, Chernyavsky AM and Ragino YI (2018) Dynamics of Changes in Concentration of Inflammatory and Destructive Biomarkers at Various Stages of Atherosclerotic Plaque Development. Cardiac Sci Res $J$ Volume 1(2): 1-5 